\title{
Importancia de las imágenes radiológicas para el diagnóstico de la condrocalcinosis
}

\author{
F. de la Guía G alipienso, G. VÁZquez Perfecto*, B. Pérez Villacastín ** \\ Especialista en Medicina Familiar y Comunitaria. ${ }^{*}$ Residente en Medicina \\ Familiar y Comunitaria. ${ }^{* * E s p e c i a l i s t a ~ e n ~ R a d i o d i a g n o ́ s t i c o . ~ F u n d a c i o ́ n ~ J i m e ́-~}$ \\ nez Díaz. \\ Universidad Autónoma de Madrid
}

\section{RESUMEN}

La condrocalcinosis consiste en el depósito de cristales de pirofosfato cálcico dihidratado en los fibrocartílagos y en el cartílago hialino articular. La liberación de estos cristales al medio sinovial desencadena una artritis aguda muy parecida a la gotosa, por lo que se también se denomina pseudogota.

Presentamos el caso de un varón de 38 años con lumbalgia intensa irradiada hacia región glútea y cara posterior del muslo izquierdo, con dolores en varias articulaciones, signos inflamatorios e impotencia funcional, con brotes de 10 días de duración, e intervalos libres de síntomas, con una duración total de 2 años.

Palabras clave: Condrocalcinosis. Pseudogota. Radiología. Hipomagnesemia.
Essential role of radiological images in the diagnosis of chondrocalcinosis

\begin{abstract}
Deposit of dihydrate calcium pyrophosphate crystals on fibrous cartilage and joint hyaline cartilage is called chondrocalcinosis. Because the release of those crystals into the synovial fluid induces an acute arthritis similar to gout arthritis, the clinical syndrome is refered to as pseudogout.

Here we report a case of 38-years old man, caracterized by two years of a strong backache radiating to gluteal and posterior region of the left thigh with diffuse pain affecting several joints, inflammatory signs and joint functional disability. The patient has symptoms for ten days periods, intermittent outbreaks and intercalated non-symptomatic periods.
\end{abstract}

Key words: Chondrocalcinosis. Pseudogout. Radiology. Hypomagnesemia.

\section{INTRODUCCIÓN}

La condrocalcinosis es la enfermedad que resulta del depósito de cristales de pirofosfato cálcico dihidratado (PPCD) en los fibrocartílagos y en el cartílago hialino articular. Fueron McCarthy et al, los que a inicios de los años 60, descubrieron en el líquido sinovial cristales diferentes al ácido úrico y que estaban constituidos por PPCD, de ahí que se le conozca también co- mo enfermedad por depósito de cristales de $\mathrm{PPCD}^{1}$. La etiología es variada: a) hereditaria; b) esporádica o idiopática; y c) asociada a enfermedades metabólicas. La forma de presentación más frecuente es la de una artritis crónica progresiva con cuadros superpuestos de episodios agudos inflamatorios, y la radiología, medio al alcance de la Atención Primaria, constituye una herramienta esencial en el diagnóstico de esta enfermedad. 


\section{OBSERVACIÓN CLÍNICA}

Presentamos el caso de un varón de 38 años de edad que consulta por presentar de forma aguda un dolor lumbar intenso con irradiación hacia la región glútea y cara posterior del muslo izquierdo, hasta el hueco poplíteo, con dolores en distintas articulaciones (sacroilíacas, rodillas, muñecas, codos, hombro, nuca y tobillos). Destaca la presencia de signos inflamatorios, tanto en articulaciones pequeñas como grandes, con importante impotencia funcional que le dificulta su tarea diaria, así como el mal estado general y la febrícula ( $38^{\circ}$ máximo). Al insistir en la presentación de los síntomas, el paciente indica que esta sintomatología la viene presentando desde hace más de 2 años, con brotes con una duración no superior a los 10 días, e intervalos libres de síntomas. Tiene antecedentes de brucelosis (4 años antes a esta consulta), con aglutinaciones negativas en el momento de la consulta. No refiere ningún antecedente familiar de artropatía.

Ante este cuadro clínico, se le realiza estudio radiográfico (Figs. 1 y 2) de las articulaciones afectas, comprobándose una calcificación lineal bilateral en ambos fibrocartílagos articulares de las rodillas, con la calcificación del cartílago hialino de ambos cóndilos femorales. Del mismo modo, se observa la calcificación lineal del cartílago de la sínfisis pubiana, así como presencia de finas calcificaciones de cartílago articular en ambos codos, con calcificacio nes irregulares en la bolsa sinovial del olécranon.

Ante los hallazgos radiográficos mencionados, se practicó estudio analítico, objetivándose normalidad de todos los parámetros bioquímicos, excepto una hipomagnesemia hipersecretora de origen tubular renal [(magnesio en sangre: 0,90 (cn: 2,0 \pm $0,2 \mathrm{mg} / \mathrm{dl}$ ); magnesio intraeritrocitario: 0,1 ( $\mathrm{cn}: 4,5$ $\pm 0,5 \mathrm{mg} / 100 \mathrm{mgHg}$ ); eliminación urinaria de magnesio: 300 (cn: $35-150 \mathrm{mg} / 24 \mathrm{~h}$ ); índice de excreción de $\mathrm{Mg}$ : 0,089 (cn: 0,025 0 0,100 mg/100 GFR)]; hipopotasemia (potasio plasmático: 2,9 $\mathrm{mEq} / \mathrm{l}$; eliminación urinaria de potasio: 50 $\mathrm{mEq} / 24 \mathrm{~h}$ ) y alcalosis metabólica ( $\mathrm{pH}$ arterial: 7,45 ; $\mathrm{PCO}_{2}: 42 \mathrm{mmHg}$; bicarbonato: 28,7$)$.

La clínica que presentaba el paciente, los hallazgos radiológicos y analíticos, así como la presencia de cristales de pirofosfato cálcico en la biopsia de líquido sinovial que se le practicó posteriormente, permitieron realizar el diagnóstico de condrocalcinosis con hipomagnesemia hipersecretora.

El cuadro clínico mejoró ostensiblemente con la administración oral de óxido de magnesio y cloruro potásico, con normalización de los valores sanguíneos y proporcionado aumento de su excreción

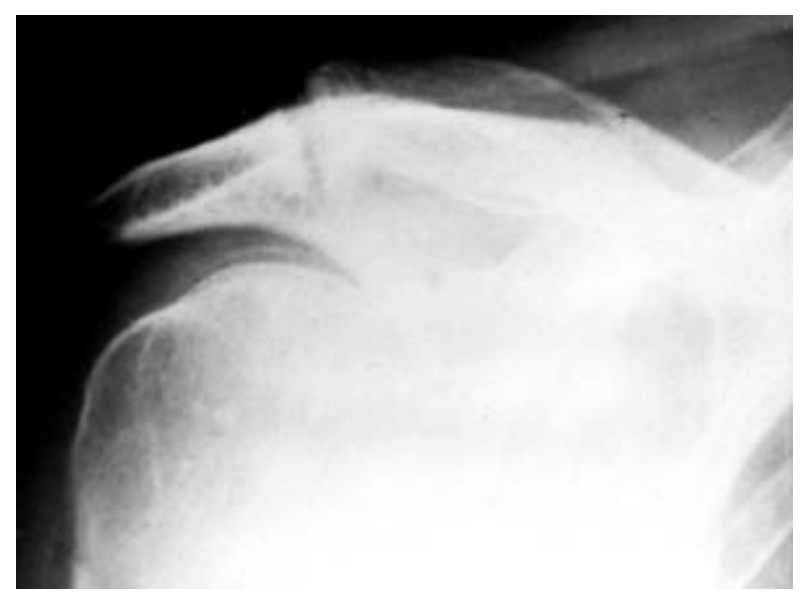

Figura 2

Proyección anteroposterior de hombro derecho. Presencia de calcificación lineal en la interlínea articular

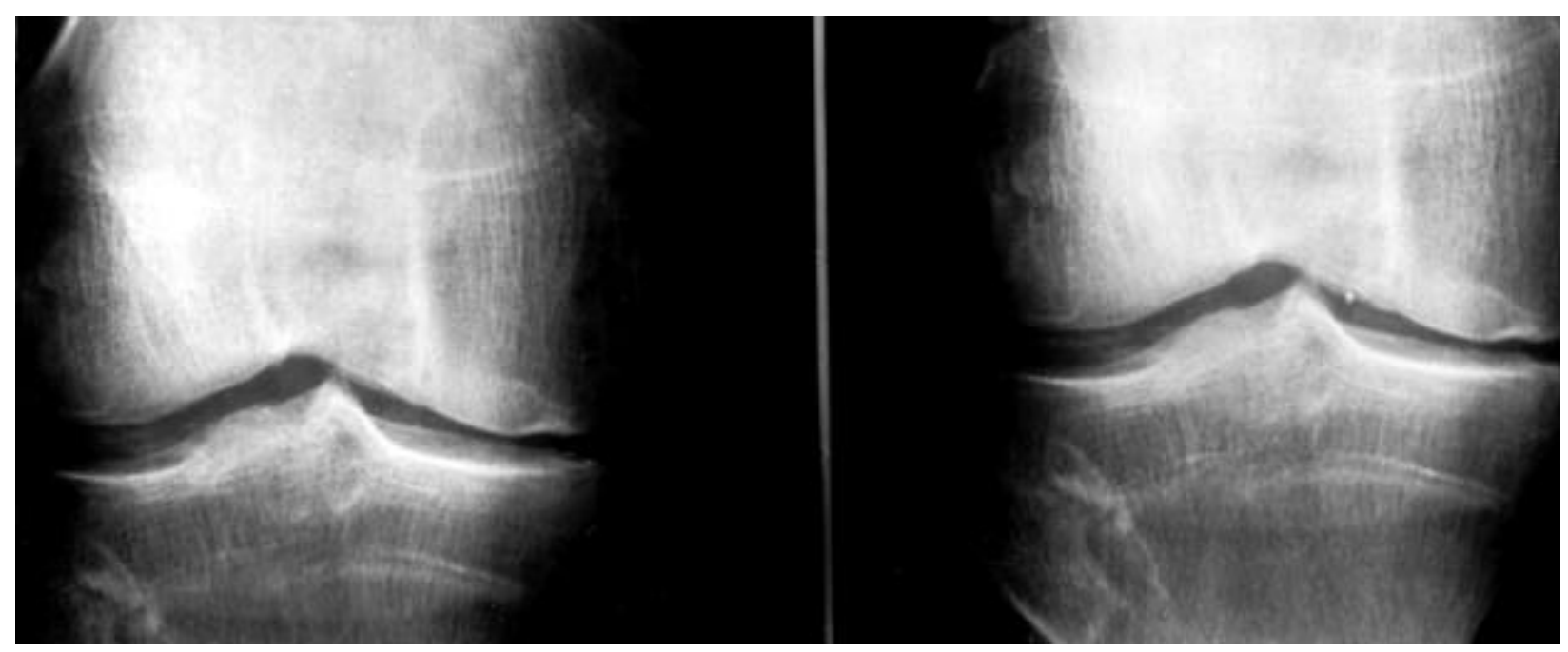

Figura 1

Proyección anteroposterior de rodilla. Se visualizan imágenes lineales, de densidad calcio, correspondientes a calcificación del cartígalo hialino 
fraccional en orina, confirmando el origen tubular renal de dicha alteración metabólica. Fue tratado con antiinflamatorios no esteroideos, tales como el diclofenaco y el aceclofenaco (actualmente con $200 \mathrm{mg} /$ día), cediendo los signos inflamatorios y la sintomatología dolorosa que padecía.

\section{COMENTARIOS}

La condrocalcinosis o enfermedad por depósito de cristales de pirofosfato cálcico dihidratado es una entidad infradiagnosticada en nuestro medio. La presencia de esta enfermedad aumenta con la edad y es frecuentemente asintomática, motivos por los cuales no es tomada en cuenta en muchas ocasiones. El cuadro clínico es muy parecido al de la artritis gotosa, y por ello también se le denomina pseudogota ${ }^{2}$. Puede tener diversas etiologías: a) hereditaria, que se ha descrito en familias eslovacas, chilenas, canadienses, japonesas, holandesas, francesas y españolas (por ejemplo, en familias cántabras y alrededores de Madrid); en la mayoría de estos grupos la enfermedad es grave y de comienzo temprano, se heredan principalmente en forma autosómica dominante, y constituyen un grupo heterogéneo en cuanto a las manifestaciones clínicas, fenotipos, edad de comienzo o tipo de herencia. Serán muy importantes los estudios genéticos para detectar los posibles defectos responsables de la enfermedad; b) esporádica o idiopática, que es la observada habitualmente, sobre todo a partir de los 60 años (hasta en un 30\% en las personas con más de 80 años), y que puede estar muy ligada en sus mecanismos a las formas familiares, tal y como se ha comprobado en estudios realizados en Madrid, donde un $38 \%$ de las personas con condrocalcinosis aparentemente esporádicas son de tipo familiar ${ }^{3}$; y c) asociada a enfermedades metabólicas (Tabla I), como el hiperparatiroidismo primario, donde la condrocalcinosis ha sido observada en un 20-30\% de estos pacientes; la hipomagnesemia (como en el caso que exponemos); la hemocromatosis (alrededor de la mitad de estos pacientes presentan depósito de cristales de pirofosfato cálcico dihidratado en las rodillas, muñecas y otras articulaciones) o la amiloidosis 4 .

La condrocalcinosis afecta por igual a ambos sexos (discreta preferencia por las mujeres en las formas esporádicas), sin predilección racial y a pacientes en edades medias y avanzadas de la vida, siendo extremadamente infrecuente encontrar alteraciones en pacientes con menos de 20 años. La articulación que con más frecuencia se ve afectada es la rodilla, siendo también habitual la afectación de muñecas, sínfisis pubiana, codos y hombros. La forma de presentación más frecuente (35-60\%) es la de una artritis crónica progresiva con cuadros su-
Tabla I

ENTIDADES ASOCIADAS CON LA ENFERMEDAD POR DEPÓSITO DE CRISTALES DE PIROFOSFATO

GRUPO A

Asociación verdadera. Alta probabilidad

1. Hiperparatiroidismo primario

2. Hipocalciuria hipercalcémica familiar

3. Hemocromatosis

4. Hemosiderosis

5. Hipofosfatasia

6. Hipomagnesemia

7. Síndrome de Bartter

8. Hipotiroidismo

9. Gota

10. Osteoartropatía neuropática

11. Amiloidosis

a) Traumatismo localizado

b) Cirugía por osteocondritis disecante

12. Síndrome de hipermovilidad

13. Tratamiento prolongado con corticoides

14. Edad avanzada

\section{GRUPO B}

Asociación verdadera. Moderada probabilidad

1. Hipertiroidismo

2. Litiasis renal

3. Hiperostosis esquelética difusa idiopática

4. Ocronosis

5. Enfermedad de Wilson

6. Artritis hemofílica

GRUPO C

Asociación improbable

1. Diabetes mellitus

2. Hipertensión

3. Azoemia

4. Hiperuricemia

5. Ginecomastia

6. Artritis reumatoide

7. Enfermedad ósea de Paget

8. Acromegalia

Tomado de: McCarthy DJ. Ann Rheum Dis 1983; $42: 423$

perpuestos de episodios agudos inflamatorios (pseudogota), que suele afectar a las articulaciones mencionadas de forma simétrica y bilateral, ocasionando una precipitación de cristales de pirofosfato cálcico en el seno de la articulación. En estos casos el diagnóstico puede verificarse mediante la extracción del líquido sinovial en el que se demuestran los cristales de pirofosfato, libres o incluidos en los leucocitos, que observados con luz polarizada tienen una birrefringencia débilmente positiva y un eje de extinción inclinado, mientras que los cristales de urato monosódico poseen una intensa birrefringencia negativa con la luz polarizada compensada y su eje de extinción es axial. 
Tabla II

\section{CRITERIOS DIAGNÓSTICOS}

I. Demostración de cristales de pirofosfato cálcico en la biopsia, en la necropsia o en el aspirado de líquido sinovial, por difracción de los rayos $X$ o mediante análisis químico.

II. A) Identificación de cristales sugestivos de pirofosfato cálcico en el líquido sinovial por medio del microscopio de luz polarizada (con birrefringencia débilmente positiva o nula).

B) Presencia de calcificaciones típicas en la radiología (calcificación de los meniscos, sínfisis del pubis, ligamentos triangulares del carpo y cartílago articular, en este caso en forma de una delgada línea paralela a la superficie articular).

III. A) Artritis aguda, especialmente de articulaciones grandes, con hiperuricemia concomitante o sin ella. B) Artropatía crónica, en especial de rodillas, caderas, carpos, codos, hombros y metacarpofalángicas, a veces acompañada de ataques agudos.

De acuerdo con estos criterios, la enfermedad se divide en tres categorías:

A) Definida: Ha de cumplir el criterio I o bien los criterios IIA y IB.

B) Probable: Ha de cumplir el criterio IIA o bien el IIB.

C) Posible: Criterio IIIA, o bien el IIIB. Esta categoría no es diagnóstica y su único valor es el de alertar al médico sobre la posibilidad de una pseudogota.

Tomado de: McCarthy DJ. Dis of the Month 1994; 6: 253-300

En la forma crónica, que es la que presentamos, se establecen manifestaciones articulares persistentes, y el diagnóstico depende fundamentalmente de la radiología (Tabla II) ${ }^{5}$. Es necesario indicar que el solo hallazgo radiográfico de condrocalcinosis no permite asegurar que la sintomatología es una consecuencia directa de los depósitos de cristales de pirofosfato cálcico ${ }^{4}$, pero la importancia de los hallazgos radiológicos radica en que permiten formular un diagnóstico de presunción muy orientativo y fidedigno en una consulta de Atención Primaria, ya que la radiografía anteroposterior de rodilla identifica el $90 \%$ de los casos y junto con la de pelvis y manos puede detectar la totalidad de los pacientes con condrocalcinosis. En la tabla II se describen los criterios diagnósticos, de tal modo que la presencia de calcificaciones típicas en la radiología, que a continuación describimos, junto a la presencia de cristales sugestivos de pirofosfato cálcico en el líquido sinovial por microscopio de luz polarizada son necesarios para diagnosticar de forma definida esta entidad.

Los hallazgos radiológicos (Figs. 1 y 2) más significativos son: a) calcificación del cartílago (condrocalcinosis) que puede comprometer el fibrocartílago y el cartílago hialino. Los depósitos fibrocartilaginosos aparecen como áreas radiodensas muy irregulares, de aspecto puntiforme, en el interior de la cavidad articular, mientras que las calcificaciones del cartílago hialino son finas, lineales y paralelas al hueso subcondral subyacente, así el aspecto radiográfico de las calcificaciones del menisco y del cartílago articular es muy característico; b) calcificación de la membrana sinovial, localizada en los márgenes de la articulación, que adopta un aspecto más irregular; c) calcificación de la cápsula articular; y d) calcificaciones de estructuras ligamentosas, tendinosas, vasculares o tejidos blandos.

La rodilla es la articulación principalmente afectada (y más típicamente los meniscos) desde el punto de vista clínico y radiológico, siendo también frecuente la afectación del ligamento triangular del carpo y los fibrocartílagos de la sínfisis del pubis, de ahí la importancia de realizar una radiografía de rodilla, mano y pelvis a todo paciente en el que se tenga la sospecha de esta enfermedad. También pueden verse afectadas las articulaciones tibiotarsianas, los codos y los hombros. Una de las articulaciones que se afecta infrecuentemente es la primera metatarsofalángica.

Clínicamente la forma crónica puede manifestarse simulando una artritis reumatoide o una artrosis, o bien puede ser asintomática y descubrirse a través de un examen radiológico por otros motivos. El estudio radiográfico puede proporcionar una evidencia definitiva de depósito de PPCD en estadios relativamente tempranos de la enfermedad, ya que ante estados avanzados de la enfermedad puede ser difícil la visualización de estos hallazgos por la destrucción del cartílago articular, considerándose un test de screening muy valioso la realización de una radiografía de ambas rodillas, muñecas y sínfisis del pubis ${ }^{5-7}$.

La presentación de una condrocalcinosis con hipomagnesemia está apoyada en el hecho de que la actividad de la enzima pirofosfatasa depende de la presencia del ión magnesio, existiendo una relación directa entre la solubilidad del pirofosfato cálcico y la concentración de magnesio ${ }^{8}$.

En general, el tratamiento es básicamente sintomático y tiene como misión mantener la capacidad funcional de la articulación y mitigar las crisis agudas. Los objetivos del tratamiento de base son la eliminación de la sobrecarga con adaptación de las actividades diarias, corrección de las posibles deformidades, y empleo de analgésicos o antiinflamatorios, que deben ser empleados con precaución en ancianos por sus efectos secundarios y patologías asociadas. La administración de magnesio puede inducir una regresión de los depósitos de pirofosfato y de la sintomatología acompañante (tal y 
Más información o envíos a: $\ulcorner\quad$ EDCLONSSA

Castelló, 128 - 28006 Madrid

Telf. 91-7820034 - Fax: 91-5615787

e-mail: suscripc@grupoaran.com

http://www.arannetworks.es 
como sucede con el paciente que describimos), sin embargo, en aquellas formas que son secundarias, por ejemplo, a hemocromatosis e hiperparatiroidismo primario, el tratamiento de la enfermedad de base no induce una disminución de estos depósitos, ni a una mejora en la artritis ${ }^{9}$. En casos muy agudos y severos, si la articulación lo permite, puede ser útil la aspiración completa del líquido sinovial con inyección local intraarticular de corticoides ${ }^{10}$. El reemplazo articular es el método más efectivo para suprimir el dolor y restablecer la función articular en aquellos pacientes que presenten signos de osteoartritis grave con destrucción articular ${ }^{4}$.

En conclusión, con los medios disponibles actualmente en las consultas de la mayoría de los médicos de familia, y con la ayuda de una radiografía de rodilla, mano y pelvis, puede ser suficiente para sospechar la enfermedad por depósitos de cristales de pirofosfato cálcico en el cartílago, fibrocartílago o menisco articular, e iniciar un estudio diagnóstico para determinar la etiología de esta entidad.

\section{CORRESPONDENCIA}

Fernando de la Guía Galipienso

C/ Blasco de Garay, 54- $1^{\circ} \mathrm{B}$

28015 Madrid

\section{Bibliografía}

1. McCarthy DJ. Calcium pyrophosphate dihydrate crystal deposition disease (pseudogout syndrome). Clinical aspects. Clin Rheum Dis 1977; 3: 61-89.

2. Snaith ML. ABC of rheumatology. Gout, hyperuricaemia, and crystal arthritis. BMJ 1995; 310: 521-4.

3. Fernández-Dapica MP, Reginato AJ. Familiar condrocalcinosis in Spain. Arthritis Rheum 1992; 35:45.

4. Reginato AJ. Artropatía por cristales de pirofosfato cálcico. Capítulo 9.4. En: Pascual E, Rodríguez V, Carbonell J, Gómez-Reino JJ. Tratado de Reumatología (Tomo II). Madrid: Arán Ediciones, 1998; 1477 95.

5. Resnick D, Niwayama G. Calcium pyrophosphate dihydrate (CCPD) crystal deposition disease. In: Saunders WB, ed. Diagnosis of bone and joint disorders. Philadelphia: Saunders Company, 1995; 1, 1556-614.
6. Steinbach LS, Resnick D. Calcium pyrophosphate dihydrate crystal deposition disease revisited. Radiology 1996; 200: 1-9.

7. Zitman D, Sitaj S. Chondrocalcinosis articularis: clinical and radiological study. Ann Rheum Dis 1996; 55: 30-3.

8. Zarco P, Jaller JJ, Rapado A, Herrero-Beamont G, Traba ML, Grant C, et al. Estudios metabólicos en dos casos de condrocalcinosis con hipomagnesemia. Rev Clin Esp 1989; 185: 77-81.

9. Jones AC, Chuck AJ, Aire EA, Green DJ, Doherty M. Diseases associated with calcium pyrophosphate deposition disease. Seminar Arthritis Rheum 1992; 22: $188-202$.

10. Tornero J, Vidal J. Infiltraciones en la patología de la rodilla. Medifam 2000; 10: 178-87. 